\title{
Correction: A new strategy for membrane-based direct air capture
}

\author{
Shigenori Fujikawa (1) - Roman Selyanchyn (1) - Toyoki Kunitake
}

Published online: 18 November 2020

(c) The Society of Polymer Science, Japan 2020

Correction to: Polymer Journal

https://doi.org/10.1038/s41428-020-00429-z

The authors noted that the grant numbers in Acknowledgement were not correct. The grant number of the Japan Society for Promotion of Science (JSPS) for a Grant-in-Aid for Scientific Research (B) and for a Grant-in-Aid for Early
Career Scientists were originally given as ' $20 \mathrm{H} 02781$ ' and '19K153427, respectively. Each grant number should be 'JSPS KAKENHI Grant Number JP20H02781' and 'JSPS KAKENHI Grant Number JP19K15342' for a Grant-in-Aid for Scientific Research (B) and for a Grant-in-Aid for Early Career Scientists, respectively. The original article has been corrected. 